\title{
Bioatividade de nanoformulações de nim sobre a traça-do-tomateiro
}

\author{
Bioactivity of neem nanoformulations on tomato pinworm
}

\author{
Fátima Teresinha Rampelotti Ferreira' ${ }^{\mathrm{I}}$ José Djair Vendramim ${ }^{\mathrm{II}}$ Moacir Rossi Forim ${ }^{\mathrm{III}}$
}

\section{RESUMO}

\begin{abstract}
O uso de plantas inseticidas é uma importante ferramenta para o manejo de insetos-praga. Visando ao controle de Tuta absoluta (Meyrick) (Lepidoptera: Gelechiidae), nanoformulações de nim foram avaliadas. Após a estimativa de uma $C L_{50}$ para uma formulação de óleo comercial de nim, bioensaios de seleção foram realizados com 22 nanoformulações. Para isso, lagartas neonatas foram alimentadas sobre folíolos tratados com soluções das nanoformulações durante 10 dias. Com as duas nanoformulações mais promissoras, NC40 aquoso e NC40 pó (NC 40=nanocápsulas de Poli- $\beta$-hidroxibutirato), foi avaliado o efeito sobre o desenvolvimento e a longevidade do inseto. A $C L_{50}$ para o óleo de nim foi estimada em $0,20 \%$ ou $1,31 \mathrm{mgL}^{-1}$ de azadiractina. As nanoformulações NC40 aquoso e NC40 pó afetaram o desenvolvimento do inseto.
\end{abstract}

Palavras-chave: Tuta absoluta, Azadirachta indica, Meliaceae, planta inseticida.

\section{ABSTRACT}

The use of insecticide plants is an important tool in the management of insect pests. Aiming to control Tuta absoluta (Meyrick) (Lepidoptera: Gelechiidae), neem nanoformulations were evaluated. After estimating the $L C_{50}$ for a commercial neem oil formulation, selection bioassays were performed with 22 nanoformulations. In order to do that, newly emerged caterpillars were fed on leaflets treated with nanoformulation solutions for 10 days. The effect on the development and longevity of the insect was evaluated with the two most promising nanoformulations, aqueous NC40 and powdered NC40 (NC 40 = Poly- -hydroxibutirate nanocapsules). The $L C_{50}$ for neem oil was estimated in $0.20 \%$ or $1.31 \mathrm{mgL}^{-1}$ of azadiractin. The nanoformulations aqueous NC40 and powdered NC40 affected the insect development.

Key words: Tuta absoluta, Azadirachta indica, Meliaceae, plant insecticides.

\section{INTRODUÇÃO}

A traça Tuta absoluta (Meyrick) (Lepidoptera: Gelechiidae) é uma das principais pragas do tomateiro, causando sérios prejuízos devido aos danos diretos e indiretos que ocasiona à cultura durante todo o seu ciclo (DESNEUX et al., 2010). O difícil controle e os grandes prejuízos têm levado à frequente aplicação de inseticidas (PICANÇO et al., 1995), razão pela qual novas estratégias integradas ao controle químico têm sido buscadas para diminuir o número de pulverizações, a seleção de populações resistentes e os problemas de contaminação ambiental e dos operadores.

Nesse contexto, plantas inseticidas podem ser uma alternativa viável. As pesquisas visando à utilização de extratos vegetais para controle de $\boldsymbol{T}$. absoluta iniciaram-se na década de 90, com a constatação do efeito de meliáceas sobre o inseto (FERRACINI et al., 1991, 1993). Mais recentemente, foram realizados trabalhos avaliando o efeito de extratos de Trichilia spp. (VENDRAMIM \&

IFaculdade Centro-Matogrossense (FACEM), 78556-268, Sinop, MT, Brasil. E-mail: ftrampelotti@hotmail.com. Autor para correspondência.

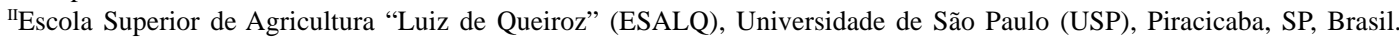

IIUniversidade Federal de São Carlos (UFSCar), São Carlos, SP, Brasil. 
THOMAZINI, 2001; CUNHA et al., 2005; 2006), de Melia azedarach L. (BRUNHEROTTO et al., 2010) e de Azadirachta indica A. Juss. (GONÇALVESGERVÁSIO \& VENDRAMIM, 2004; 2007).

Embora o efeito de plantas inseticidas já tenha sido comprovado para diversas pragas, a baixa estabilidade, a degradação e a concentração insuficiente dos ingredientes ativos nos extratos vegetais são apontadas como limitações para o seu uso pelos produtores. Dentro das perspectivas para desenvolvimento de moléculas estáveis e com alto efeito sobre organismos alvo, a nanotecnologia tem se apresentado como importante ferramenta, considerando sua aplicação no desenvolvimento de produtos contendo ingredientes ativos em escala nanométrica. A utilização da nanotecnologia no desenvolvimento de partículas agrotóxicas apresenta vantagens pela sua maior estabilidade e menor contaminação do ambiente. Assim, a nanotecnologia pode ser aplicada na obtenção de materiais funcionais por meio do controle da matéria na escala de nanômetros, implicando sistemas que apresentem novos fenômenos e propriedades (DURÁN \&AZEVEDO, 2007), garantindo maior estabilidade dos compostos provenientes de plantas inseticidas, aumentando seu potencial de controle e o seu efeito residual.

O nim (Azadirachta indica) é uma das plantas mais estudadas em todo mundo e, devido a suas características inseticidas sobre diferentes grupos de artrópodes, tem-se avaliado sua aplicação sobre insetos-praga (MARTINEZ, 2002). Diante disso, o desenvolvimento de uma formulação estável otimizaria a pulverização desse bioinseticida, caracterizando um insumo ambientalmente correto e reduzindo o número de pulverizações com inseticidas sintéticos, necessárias para controle do inseto. Dessa forma, o objetivo deste trabalho foi avaliar a bioatividade de 22 nanoformulações de nim sobre T. absoluta.

\section{MATERIAL E MÉTODOS}

Obtenção dos insetos e das plantas de tomateiro Os insetos utilizados foram obtidos de criação em hospedeiro natural mantida no laboratório de Plantas Inseticidas da ESALQ/USP. As condições de criação e manutenção dos bioensaios foram $25 \pm 2^{\circ} \mathrm{C}$; $50 \pm 10 \%$ UR; fotofase: $14 \mathrm{~h}$. Plantas de tomateiro da cultivar Santa Clara foram cultivadas em casa de vegetação. As folhas dessas plantas foram utilizadas para a criação dos insetos e, também, nos bioensaios.

\section{Estimativa da $\mathrm{CL}_{50}$}

A concentração letal $\left(\mathrm{CL}_{50}\right)$ foi estimada para a formulação de óleo de nim Organic Neem ${ }^{\circledR}$ com teor inicial de azadiractina de 773mg L ${ }^{-1}$ (FORIM et al., 2010). Avaliaram-se as concentrações de $0 ; 0,015 ; 0,03 ; 0,06$; 0,12; 0,24 e 0,48\% do óleo dissolvido em água destilada, sendo a pulverização sobre as folhas de tomateiro feita com auxílio de uma pistola tipo gravidade (Arprex ${ }^{\circledR}$ modelo 5, 20/30lb pol-2 com bico de 0,8mm) adaptada como microatomizador. Após a eliminação do excesso de umidade, os folíolos tiveram o pecíolo envolvido por algodão hidrófilo umedecido e foram colocados nas unidades experimentais (UE) (placa plástica de $6 \mathrm{~cm}$ de diâmetro x 2cm de altura). Sobre o folíolo tratado, foram colocadas quatro lagartas neonatas de $\boldsymbol{T}$. absoluta. O delineamento experimental foi inteiramente casualizado com sete tratamentos e quatro repetições (sete UE/repetição). As avaliações de mortalidade larval foram diárias, até o 10ํ dia após o tratamento.

Bioensaios de seleção das nanoformulações de nim

Vinte e duas nanoformulações foram testadas em quatro bioensaios. As nanoformulações testadas foram obtidas junto ao Laboratório de Produtos Naturais da UFSCar/SP. Com base na $\mathrm{CL}_{50}$, prepararam-se soluções de cada nanoformulação. Os procedimentos para pulverização, montagem das UE e delineamento foram iguais ao descrito no bioensaio de estimativa da $\mathrm{CL}_{50}$

Após esses bioensaios, foram selecionadas as nanoformulações NC40 aquoso e NC40 pó (NC40 = nanocápsulas de poli- -hidroxibutirato). Essas nanoformulações e o óleo de nim comercial foram avaliados quanto ao efeito sobre o desenvolvimento e longevidade de T. absoluta.

Efeito sobre o desenvolvimento e a longevidade

Os tratamentos foram pulverizados sobre folhas de tomateiro através de uma pistola tipo gravidade. Os demais procedimentos foram iguais ao descrito no bioensaio de estimativa da $\mathrm{CL}_{50}$. Avaliações diárias foram realizadas para observação da mortalidade até que as lagartas sobreviventes pupassem, ocasião em que as pupas foram retiradas das folhas e individualizadas em tubos de vidro tamponados com algodão. Foram avaliados os seguintes parâmetros: duração das fases larval e pupal, peso de pupas (24h), período de L1 (lagarta neonata) a adulto e longevidade. O delineamento experimental foi inteiramente casualizado com três tratamentos + controle água em quatro repetições (sete UE/repetição).

\section{Análise estatística}

Para a estimativa de $\mathrm{CL}_{50}$ do óleo de nim, foi utilizada a análise de probit no programa PoloPlus 1.0 (LEORA SOFTWARE, 2003). Para os demais bioensaios, a normalidade e homocedasticidade foram 
aferidas pelos testes de Shapiro Wilk e Bartlett. Quando os dados não apresentaram distribuição normal, foi utilizado o teste não paramétrico Kruskal-Wallis (KRUSKAL \& WALLIS, 1952). O teste de Tukey ou de comparação múltipla de Kruskal-Wallis foi utilizado para o contraste de médias. O software estatístico R foi utilizado para essas análises (PROGRAMAR, 2008). A análise de sobrevivência de Kaplan-Meier foi feita com o uso do software SPSS (SPSS, 2008), com comparações pareadas pelo teste de log-rank (Mantel-Cox) a $\mathrm{P}<0,05$ para verificar as diferenças entre os grupos de tratamentos (KAPLAN \& MEIER, 1958).

\section{RESULTADOS E DISCUSSÃO}

Estimativa da $\mathrm{CL}_{50}$ do óleo de nim

$\mathrm{A} \mathrm{CL}_{50}$ estimada para o óleo de nim(Organic Neem $^{\circledR}$ ) para lagartas de T. absoluta foi de $0,20 \%$, com coeficiente angular de $2,10 \pm 0,30, \chi^{2}=2,74$, heterogeneidade de 0,69 e 4 graus de liberdade. Com base na quantificação de azadiractina, feita no início do bioensaio, a concentração de $0,20 \%$ corresponde a 1,31 $\mathrm{mgL}^{-1}$ de azadiractina. Marcomini (2009), utilizando a mesma formulação de óleo de nim, estimou a $\mathrm{CL}_{50}$ de $0,74 \%$ em dieta artificial e $0,64 \%$ em plantas de milho para Spodoptera frugiperda (J. E. Smith) (Lepidoptera:
Noctuidae). Essa diferença observada entre espécies de insetos se deve, ao menos em parte, ao tamanho das lagartas, demonstrando a importância das estimativas de $\mathrm{CL}_{50}$ para a espécie em estudo.

Seleção das nanoformulações mais promissoras No primeiro bioensaio (Tabela 1), a maior mortalidade foi observada na nanoformulação de cápsulas de PHB (NCL13), a qual não diferiu do óleo de nim, mas foi superior aos valores registrados no controle água e nos demais tratamentos, com exceção das nanoformulações de cápsulas de PCL/PHB (NCL14) e de PCL (NCL12), que propiciaram mortalidades intermediárias, não diferindo de nenhum dos tratamentos. Nos tratamentos NCL15, NCL16 e NCL17, que não continham nim, observaram-se mortalidades inferiores às registradas para as nanoformulações equivalentes contendo nim. Os referidos tratamentos causaram mortalidades similares àquela observada no controle água, demonstrando a inocuidade dos polímeros PCL e PHB utilizados nas nanoformulações sobre o inseto.

Para as nanoformulações testadas no segundo bioensaio (Tabela 1), os tratamentos propiciaram mortalidades inferiores à observada para o óleo de nim, as quais, por outro lado, não diferiram do valor constatado no controle água.

Tabela 1 - Média \pm erro padrão da mortalidade de lagartas de Tuta absoluta alimentadas, durante 10 dias, com folíolos de tomateiro tratados com nanoformulações de nim em cápsulas e esferas de PCL e PHB. $25 \pm 2^{\circ} \mathrm{C}$; $50 \pm 10 \%$ UR; fotofase: $14 \mathrm{~h}$.

\begin{tabular}{|c|c|c|}
\hline Tratamentos & Descrição & Mortalidade (\%) \\
\hline \multicolumn{3}{|c|}{ 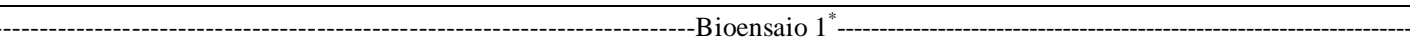 } \\
\hline NCL13 & Nanocápsulas de $\mathrm{PHB}^{1}+$ óleo/extratos de nim & $38,7 \pm 12,85$ a \\
\hline Óleo de nim & Organic Neem & $36,0 \pm 9,80$ a \\
\hline NCL12 & Nanocápsulas de $\mathrm{PCL}^{2}+$ óleo/extratos de nim & $20,7 \pm 14,53 \mathrm{ab}$ \\
\hline NCL14 & Nanocápsulas de PCL/PHB+óleo/extratos de nim & $17,1 \pm 15,00 \mathrm{ab}$ \\
\hline NCL16 & Nanocápsulas de PHB + oleato de isodecila & $13,5 \pm 10,57 b$ \\
\hline NCL17 & Nanocápsulas de PCL/PHB + oleato de isodecila & $13,5 \pm 19,44 \mathrm{~b}$ \\
\hline NCL15 & Nanocápsulas de PCL + oleato de isodecila & $12,6 \pm 7,26 \mathrm{~b}$ \\
\hline Água & Controle & $7,2 \pm 5,00 \mathrm{~b}$ \\
\hline Óleo de nim & Organic Neem & $70,3 \pm 4,56 \mathrm{~b}$ \\
\hline NSL12 & Nanoesferas de $\mathrm{PCL}^{2}+$ óleo/extratos de nim & $37,8 \pm 5,90$ a \\
\hline NSL13 & Nanoesferas de $\mathrm{PHB}^{1}+$ óleo/extratos de nim & $33,3 \pm 3,11$ a \\
\hline NSL14 & Nanoesferas de PCL/PHB + óleo/extratos de nim & $37,8 \pm 3,99$ а \\
\hline NSL15 & Nanoeferas de PCL + oleato de isodecila & $20,7 \pm 4,83$ a \\
\hline NSL16 & Nanoesferas de PHB + oleato de isodecila & $33,3 \pm 4,23$ a \\
\hline NSL17 & Nanoesferas de PCL/PHB + oleato de isodecila & $24,3 \pm 6,23$ a \\
\hline Água & Controle & $22,5 \pm 3,50$ a \\
\hline
\end{tabular}

${ }^{1}$ PHB: Poli- $\beta$-hidroxibutirato, ${ }^{2}$ PCL: Poli- $\varepsilon$-caprolactona. *Médias seguidas da mesma letra não diferem significativamente pelo teste de Tukey $(\mathrm{P}<0,05)$. ${ }^{* *}$ Médias seguidas da mesma letra não diferem significativamente pelo teste de comparação múltipla de KrusKal-Wallis $(\mathrm{P}<0,05)$ 
No terceiro bioensaio (Tabela 2), as mortalidades causadas pelos tratamentos NS38 em pó e óleo de nim em pó (OP) não diferiram dos registrados com o óleo de nim. NS38 em suspensão aquosa, R01 e R02 causaram mortalidades inferiores ao óleo de nim e superiores ao controle água. Os tratamentos NC50 e $\mathrm{PB}$, que não causaram mortalidade larval foram retirados da análise, com o intuito de melhorar a distribuição dos dados na análise estatística.

No quarto bioensaio (Tabela 2), as mortalidades causadas pela nanoformulação NC40 em suspensão aquosa e pelo óleo de nim foram semelhantes e superiores ao valor registrado para NC40 pó, sendo que esses três tratamentos diferiram do controle água.

Considerando os resultados obtidos nos quatro bioensaios, observa-se que as maiores mortalidades foram obtidas com as nanoformulações NC40 em suspensão aquosa e em pó (Tabela 2) e com a NS38 em pó (Tabela 2). Além de a mortalidade causada por essas nanoformulações ter sido maior do que no controle água, seus respectivos controles (nanoformulações contendo apenas os polímeros PHB ou PMMA) não afetaram a sobrevivência larval, demonstrando sua inocuidade a T. absoluta.

Essas diferenças de mortalidade observadas entre as nanoformulações testadas podem ter ocorrido devido à forma das nanopartículas, já que foram testadas nanoformulações compostas por cápsulas e esferas, caracterizadas basicamente pela presença e ausência do núcleo oleoso, respectivamente. As nanocápsulas são menores do que as nanoesferas e o ingrediente ativo (nesse caso, a azadiractina) é liberado todo de uma vez, quando há o rompimento da cápsula que recobre o núcleo oleoso. Já, nas esferas, o ingrediente ativo fica sobreposto nas camadas do polímero, sendo liberado gradualmente, à medida que é degradado. Assim, relacionando os resultados encontrados com a nanopartícula presente nas diferentes nanoformulações e considerando a inocuidade do polímero, pode-se inferir que tanto as nanocápsulas quanto as nanoesferas contendo nim afetaram T. absoluta.

Comparando-se a nanoformulação NC40 em suspensão aquosa e em pó, observa-se maior mortalidade na suspensão aquosa. Considerando que a eficiência de encapsulamento não atinge 100\%, moléculas de azadiractina permanecem livres na suspensão e podem afetar o inseto. Isso explicaria o menor efeito da nanoformulação em pó, em que, durante o processo de secagem, a maior parte da azadiractina livre é perdida. Em contrapartida, uma nanoformulação em pó pode ser mais estável durante o armazenamento, já que a presença de água poderia catalisar reações de hidrólise entre os componentes da nanoformulação.

Tabela 2 - Média \pm erro padrão da mortalidade de lagartas de Tuta absoluta alimentadas, durante 10 dias, com folíolos de tomateiro tratados com nanoformulações de cápsulas e esferas de PMMA, PCL, $\beta$-Ciclodextrina e PHB. $25 \pm 2{ }^{\circ} \mathrm{C}$; $50 \pm 10 \%$ UR; fotofase: $14 \mathrm{~h}$.

\begin{tabular}{|c|c|c|}
\hline Tratamentos & Descrição & Mortalidade (\%) \\
\hline NS38 & Nanoesferas de PMMA + óleo/extratos de nim + T - pó & $93,5 \pm 1,31$ e \\
\hline $\mathrm{OP}$ & Óleo comercial Organic Neem em pó & $90,7 \pm 14,16$ de \\
\hline Óleo de nim & Organic Neem & $86,6 \pm 13,17$ e \\
\hline NS38 & Nanoesferas de PMMA + óleo/extratos de nim + T - SA & $62,5 \pm 6,30 \mathrm{~cd}$ \\
\hline R01 & Extrato de óleo em pó + óleo de nim + solução $\beta$-Ciclodextrina $(a)+$ tensoativo Renex 40 & $53,7 \pm 0,54 \mathrm{~cd}$ \\
\hline $\mathrm{R} 02$ & Extrato de óleo em pó + óleo de nim + solução $\beta$-Ciclodextrina $(b)+$ tensoativo Renex 40 & $49,5 \pm 5,92$ bc \\
\hline NS40 & Nanoesferas de PCL $^{2}$ & $21,1 \pm 5,60 \mathrm{ab}$ \\
\hline PB & PMMA - solução aquosa & - \\
\hline NC50 & Nanocápsulas de PCL + oleato de isodecila & - \\
\hline Água & Controle & $2,0 \pm 0,38$ a \\
\hline NC40 & Nanocápsulas de $\mathrm{PHB}^{3}$ + óleo/extratos de nim + T - SA & $99,7 \pm 0,33$ с \\
\hline Óleo de nim & Organic Neem & $99,6 \pm 0,38$ с \\
\hline NC40 & Nanocápsulas de PHB + óleo/extratos de nim + T - pó & $71,9 \pm 7,00 \mathrm{~b}$ \\
\hline Água & Controle & $6,2 \pm 2,95$ a \\
\hline
\end{tabular}

${ }^{1} \mathrm{PMMA}$ : polimetilmetacrilato, ${ }^{2} \mathrm{PCL}$ : Poli- $\varepsilon$-caprolactona, ${ }^{3} \mathrm{PHB}$ : Poli- $\beta$-hidroxibutirato, T=Tween, SA= suspensão aquosa. Médias seguidas da mesma letra não diferem significativamente pelo teste de Tukey $(\mathrm{P}<0,05)$

Ciência Rural, v.42, n.8, ago, 2012. 
Marcomini (2009), avaliando o efeito de nanoformulações de nim em dieta artificial e em folhas de milho sobre as lagartas de $\boldsymbol{S}$. frugiperda, constatou que o uso de folhas propiciou maior eficiência, mencionando ainda que as condições ambientais podem favorecer a degradação das nanopartículas, liberando o ingrediente ativo. A nanoformulação NC40, eficiente para T. absoluta, não afetou a sobrevivência de $\boldsymbol{S}$. frugiperda, tanto em suspensão aquosa quanto em pó (MARCOMINI, 2009).

Atividade sobre o desenvolvimento

Quando as lagartas de T. absoluta foram alimentadas em folíolos tratados com as nanoformulações NC40 aquoso e NC40 pó, foi observado que a sobrevivência de lagartas diferiu entre os tratamentos ( $\chi^{2}=404,75, \mathrm{gl}=5$ e $\left.\mathrm{P}<0,001\right)$. A sobrevivência foi menor em todos os tratamentos em relação ao controle água (Figura 1 ), conforme os valores estatísticos registrados $(\mathrm{P}<0,001)$ para a comparação pareada de log rank. NC40 aquoso e o óleo de nim reduziram igualmente $(\mathrm{P}=0,758)$ a sobrevivência, enquanto NC40 pó foi menos eficiente que o óleo de nim $(\mathrm{P}<0,001)$ (Figura 1). Da mesma forma, a sobrevivência larval em NC40 aquoso foi menor do que em NC40 pó $(\mathrm{P}<0,001)$. A análise da figura 1 permite observar que tanto para a nanoformulação NC40 aquoso como para o óleo de nim a sobrevivência reduziu-se drasticamente a partir do 5o dia do início do bioensaio, atingindo $100 \%$ de mortalidade entre o $15^{\circ}$ e o $17^{\circ}$ dia. O tempo letal estimado $\left(\mathrm{TL}_{50}\right)$ para NC40 aquoso (6,7 dias) não diferiu de óleo de nim (6,8 dias), contudo, ambos foram menores do que no controle água (19,4 dias). Para NC40 pó, o $\mathrm{TL}_{50}$ foi de 14,3 dias. Cabe ressaltar que, como o tempo letal é estimado em função do tempo que cada tratamento leva para reduzir em 50\% a sobrevivência das lagartas, no controle água (em que a mortalidade larval não atingiu $50 \%$ ), o $\mathrm{TL}_{50}$ representa um valor teórico estimado pelo software utilizado.

O efeito de produtos à base de nim na sobrevivência larval de T. absoluta já havia sido demonstrado por BRUNHEROTTO et al. (2010), que testaram extrato aquoso a $0,1 \%$ de sementes de nim e por TRINDADE et al. (2000) que, utilizando o extrato metanólico de amêndoas de nim, observaram 100\% de mortalidade após quatro dias. Ressalte-se, contudo, que o efeito de nanoformulações à base de óleo de nim foi primeiramente registrado neste trabalho, com resultados similares aos obtidos com o óleo comercial de nim.

Apenas para NC40 pó obtiveram-se lagartas que completaram o desenvolvimento, verificando-se nesse tratamento alongamento da fase larval (16,1 dias) e redução do peso das pupas (23mg) em comparação ao controle água (12,2 dias e 27,9mg ,respectivamente) (Tabela 3). O alongamento da fase larval desse inseto também foi observado por BRUNHEROTTO et al. (2010)

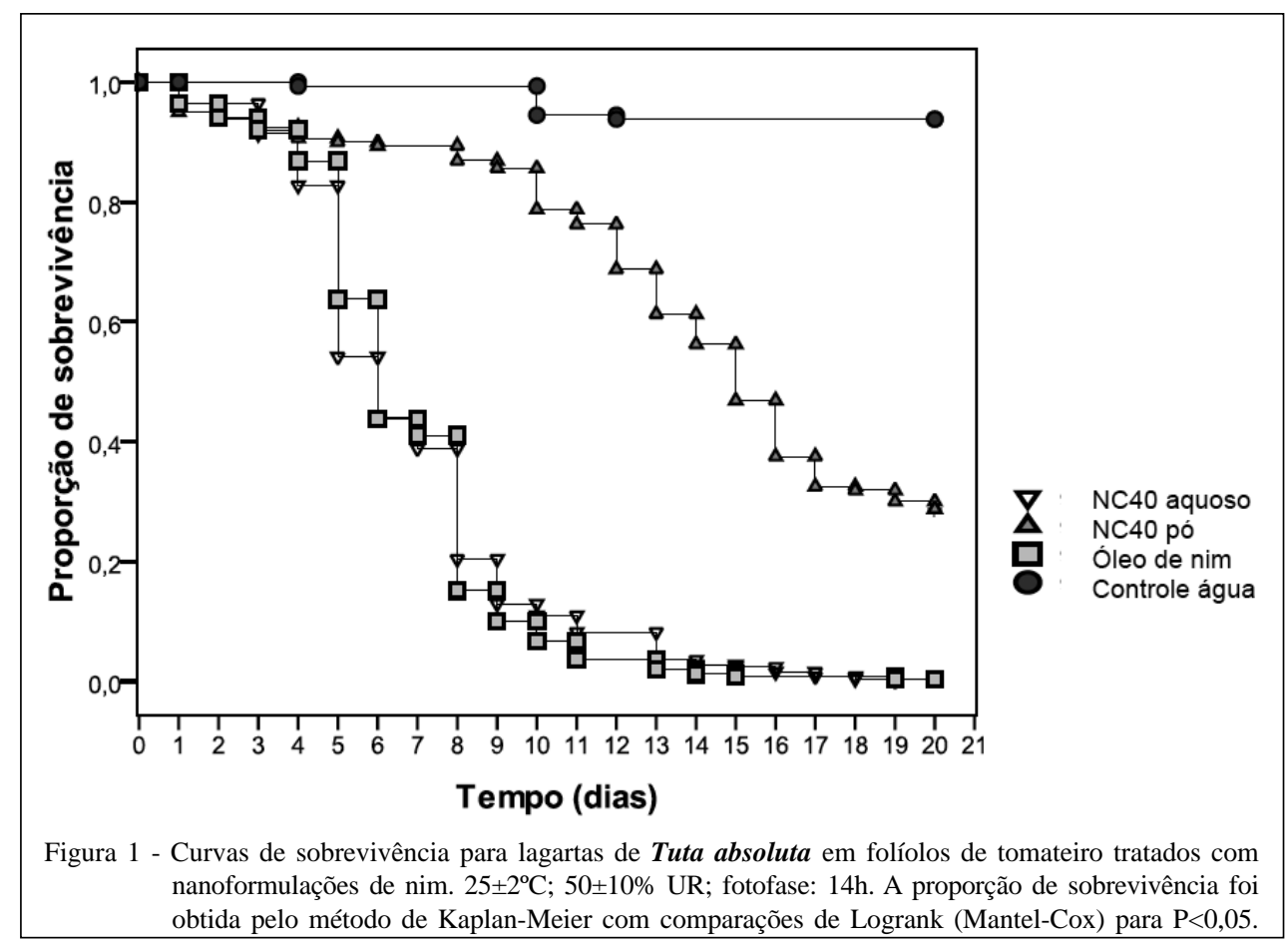

Ciência Rural, v.42, n.8, ago, 2012. 
Tabela 3 - Médias \pm erro padrão para os parâmetros duração da fase larval, peso de pupas, duração da fase pupal, período de L1 a adulto e longevidade de Tuta absoluta provenientes de lagartas alimentadas com folíolos de tomateiro tratados com nanoformulações de nim. $25 \pm 2^{\circ} \mathrm{C}$; $50 \pm 10 \%$ UR; fotofase: $14 \mathrm{~h}$.

\begin{tabular}{llllll}
\hline Tratamentos & Fase larval (dias) $^{1}$ & Peso de pupas $(\mathrm{mg})^{2}$ & Fase pupal (dias) $^{\mathrm{ns}}$ & Período L1 a adulto (dias) $^{\mathrm{ns}}$ & Longevidade (dias) $^{\mathrm{ns}}$ \\
\hline NC40-Aquoso* & - & - & - & - & - \\
NC40-Pó & $16,1 \pm 0,40 \mathrm{~b}$ & $23,0 \pm 1,38 \mathrm{~b}$ & $7,6 \pm 0,34$ & $32,9 \pm 0,29$ & $10,0 \pm 0,75$ \\
Óleo de nim* & - & - & - & - & - \\
Controle água & $12,2 \pm 0,06 \mathrm{a}$ & $27,9 \pm 0,65 \mathrm{a}$ & $7,5 \pm 0,13$ & $31,7 \pm 0,41$ & $12,1 \pm 0,36$ \\
\hline
\end{tabular}

*Tratamentos não analisados devido à ausência de dados. ${ }^{1}$ Médias seguidas da mesma letra na coluna não diferem entre si pelo teste de comparação múltipla de Kruskal-Wallis $(\mathrm{P}<0,05) .{ }^{2}$ Médias seguidas da mesma letra na coluna não diferem entre si pelo teste de Tukey $(\mathrm{P}<0,05)$. ns=não significativo

quando as lagartas foram alimentadas com folhas de tomateiro tratadas com extratos aquosos de folhas de M. azedarach e de sementes de nim.

O efeito de nanoformulações de nim sobre

T. absoluta foi primeiramente avaliado no presente trabalho, contudo, MARCOMINI (2009) já havia constatado o efeito de nanoformulações na redução do peso larval de $\boldsymbol{S}$. frugiperda.

\section{CONCLUSÃO}

Entre as 22 nanoformulações avaliadas, NCL13, NS38 pó, NS38 em suspensão aquosa, OP, R01, R02, NC40 em suspensão aquosa e NC 40 pó reduzem a sobrevivência de lagartas de T. absoluta, sendo NC40 aquoso e NC40 pó, promissoras para o controle desse inseto.

\section{AGRADECIMENTOS}

Ao Conselho Nacional de Desenvolvimento Científico e Tecnológico (CNPq), pela concessão de bolsa de estudos a primeira autora e de bolsa de produtividade em pesquisa ao segundo autor e ao Dr. Rafael Major Pitta e à Dra. Marinéia de Lara Haddad, pelo auxílio nas análises estatísticas.

\section{REFERÊNCIAS}

BRUNHEROTTO, R. et al. Efeito de genótipos de tomateiro e de extratos aquosos de folhas de Melia azedarach e de sementes de Azadirachta indica sobre Tuta absoluta (Meyrick) (Lepidoptera: Gelechiidae). Neotropical Entomology, v.39, p.784-791, 2010. Disponível em: <www.scielo.br/scielo.php?pid=S1519566X2010000500018\&script=sci_arttext $>$. Acesso em: $15 \mathrm{fev}$. 2011. doi: 10.1590/S1519-566X2010000500018.

CUNHA, U.S. et al. Potencial de Trichilia pallida Swartz (Meliaceae) como fonte de substâncias com atividade inseticida sobre a traça-dotomateiro, Tuta absoluta (Meyrick) (Lepidoptera: Gelechiidae). Neotropical Entomology, v.34, p.667-673, 2005. Disponível em: $<$ http://www.scielo.br/scielo.php?script=sci_arttext\&pid=S1519566X200500000018\&lng=en\&nrm=iso>. Acesso em: 15 fev. 2011. doi: 10.1590/S1519-566X200 5000400018.
CUNHA, U.S. et al. Frações de Trichilia pallens com atividade inseticida sobre Tuta absoluta. Pesquisa Agropecuária Brasileira, v.41, p.1579-1585, 2006. Disponível em: <http:/ /www.scielo.br/scielo.php?script=sci_arttext\&pid=S0100204X2006001100002\&lng=en\&nrm=iso $>$. Acesso em: $15 \mathrm{fev}$. 2011. doi: 10.1590/S0100-204X2006001100002.

DESNEUX, N. et al. Biological invasion of European tomato crops by Tuta absoluta: ecology, geographic expansion and prospects for biological control. Journal of Pest Science, v.83, p.197-215, 2010. Disponível em: <http:// www.springerlink.com/content/92311121j7612541/>. Acesso em: 15 fev. 2011. doi: 10.1007/s10340-010-0321-6.

DURÁN N.; AZEVEDO, M.M.M de. Rede de pesquisa em nanobiotecnologia. Disponível em: <www.comciencia.br>. Acesso em: 04 jul. 2007.

FERRACINI, V.L. et al. Controle da traça-do-tomateiro Scrobipalpula absoluta utilizando extratos vegetais. In: CONGRESSO BRASILEIRO DE ENTOMOLOGIA, 13., 1991, Recife, PE. Resumos... Recife: SEB, 1991. P.356.

FERRACINI, V.L. et al. Controle da traça-do-tomateiro Scrobipalpuloides absoluta (Lepidoptera: Gelechiidae) (Meyrick, 1917) em plantas de tomate Lycopersicon esculentum Mill) envasadas usando extratos vegetais. In: CONGRESSO BRASILEIRO DE ENTOMOLOGIA, 14., 1993, Piracicaba, SP. Resumos... Piracicaba: SEB, 1993. p.630.

FORIM, M.R. et al. Simultaneous quantification of azadirachtin and 3-tigloylazadirachtol in Brazilian seeds and oil of Azadirachta indica: application to quality control and marketing. Analytical Methods, v.2, p.860-869, 2010. Disponível em: <http://www.rsc.org/Publishing/Journals/AY/ article.asp? Journal $=$ AY39307 \& VolumeYear $=20102 \&$ Volume $=$ 2\&JournalCode $=$ AY\&MasterJournalCode $=$ AY \&SubYear=2010\&type $=$ Issue $\&$ Issue $=7 \& x=14 \& y=7 />$. Acesso em: 15 fev. 2011. doi: 10.1039/c0ay00008f.

GONÇALVES-GERVÁSIO, R.C.R.; VENDRAMIM, J.D. Modo de ação de extratos de meliáceas sobre Tuta absoluta (Meyrick, 1917) (Lepidoptera: Gelechiidae). Arquivos do Instituto Biológico, v.71, p.215-220, 2004.

GONÇALVES-GERVÁSIO, R.C.R.; VENDRAMIM, J.D. Bioatividade do extrato aquoso de sementes de nim sobre Tuta absoluta (Meyrick, 1917) (Lepidoptera: Gelechiidae) em três formas de aplicação. 
Ciência e Agrotecnologia, v.31, p.28-34, 2007. Disponível em: $<$ http://www.scielo.br/scielo.php?script=sci_arttext\&pid=S141370542007000100004\&lng=en\&nrm=iso/>. Acesso em: 15 fev. 2011. doi: 10.1590/S1413-70542007000100004.

KAPLAN, E.L.; MEIER, P. Nonparametric estimation from incomplete observations. Journal of the American Statistical Association, 53, p.457-481, 1958.

KRUSKAL, W.; WALLIS, W.A. Use of ranks in one-criterion variance analysis. Journal of the American Statistical Association, v.47, p.583-621, 1952.

LEORA SOFTWARE. Poloplus 1.0 probit and logit analysis. User's Guide. Berkeley, 2003. 36p.

MARCOMINI, A.M. Bioatividade e efeito residual de nanoformulações de nim sobre Spodoptera frugiperda (J.E. Smith). 2009. 83f. Dissertação (Mestrado em Entomologia) - Escola Superior de Agricultura “Luiz de Queiroz”, Universidade de São Paulo, SP.

MARTINEZ, S.S. (Ed). O nim Azadirachta indica: natureza, usos múltiplos, produção. Londrina: Instituto Agronômico do Paraná, 2002. 142p.
PICANÇO, M. et al. Incidência de Scrobipalpuloides absoluta em tomateiro sob diferentes sistemas de tutoramento e de controle químico. Horticultura Brasileira, v.13, p.180183, 1995.

PROGRAMA R. R development core team. R: a language and environment for (2008). Disponível em: <www.rproject.org>. Acesso em: jun. 2010.

SPSS. SPSS v.17.0.0. SPSS. Chicago, Illinois, 2008. 1 CD-ROM

TRINDADE, R.C.P. et al. Extrato metanólico da amêndoa da semente de nim e a mortalidade de ovos e lagartas da traça-dotomateiro. Scientia Agricola, v.57, p.407-413, 2000. Disponível em: <http://www.scielo.br/scielo.php?script= sci_arttext\&pid=S01030162000000300006\&lng=en\&nrm=iso/ $>$. Acesso em: 15 fev. 2011. doi: 0.1590/S01030162000000300006.

VENDRAMIM, J.D.; THOMAZINI A.P. de B.W. Traça Tuta absoluta (Meyrick) em cultivares de tomateiro tratadas com extratos aquosos de Trichilia pallida Swartz. Scientia Agricola, v.58, p.607-611, 2001. Disponível em: <http:// www.scielo.br/scielo.php?script=sci_arttext \&pid=S010390162001000300025\&lng=en\&nrm=iso/>. Acesso em: $15 \mathrm{fev}$. 2011. doi: 10.1590/S0103-90162001000300025. 